\title{
Patient led PROMs must take centre stage in cancer research
}

Roger Wilson ${ }^{1,2}$ (D)

\begin{abstract}
Plain English summary
Patient involvement in research is about adding value rather than commenting on technical quality. After 15 years as an involved patient in cancer research I started looking around and I found I was asking myself, where is the next leap in real patient benefit going to come from?

Science is making a difference to a few patients with metastatic disease but it has been achieved at a high cost. I looked at the evidence from current practices in cancer care and saw that many patients are still dying within days of taking their last chemotherapy. The ethics of current drug adoption practices and the use of new drugs are being questioned socially and politically. Evidence based practice is asking researchers to find the true value of new treatments. I came to the conclusion that quality of life assessment and patient reported outcome measures (PROMs) which capture subjective patient experience are where real and lasting progress can be made. Quality of life in research is about structured assessment. PROMs are built around the patient experience. Patient involvement is essential. The idea that you can have Patient Reported Outcomes without patient provided inputs to inform the methods and processes used, is irrational and probably unethical. In research there is a lot of scattered activity but no coherent vision for all this work. I propose a vision based on defining patient pathways informed by PROMs. Pathways would underpin a life quality description which patients can easily identify and which doctors would find easy to communicate. Pathways would also offer value to regulators and commissioners by focusing on how clinical activity delivers patient benefit.
\end{abstract}

\begin{abstract}
Patient involvement is about adding value through the application of experience and the ability to stand back and take a view, rather than commenting on technical quality. After 15 years as an involved patient in cancer research I realized that I was in the position of taking an informed view of the realities of cancer research. When I started looking around I found I was asking myself, where is the next large leap in real patient benefit going to come from? I came to the conclusion that PROs, patient reported outcomes, are where real and lasting progress can be made.

I considered current research activity and I am full of admiration for the ingenuity and scientific quality of what is being done. It is making a difference to a few of those with metastatic disease and a few more earlier in their disease experience, but it has been achieved at a high cost both financially and in terms of patient burden. I looked at the evidence from current practices in cancer care and saw that many patients are still dying within days of taking their last chemotherapy, it seems partly because of unrealistic expectations. The ethics of current drug adoption practices and the use of new drugs is being questioned socially and politically raising the challenge of finding the relative value of new treatments in terms of cost and benefit. Regulatory and HTA bodies address this through public and patient involvement (Continued on next page)
\end{abstract}

\footnotetext{
Correspondence: roger_wilson@uk2.net

${ }^{1} \mathrm{NCR}$ Consumer Forum National Cancer Research Institute, Angel Building,

London ECIV 4AD, UK

${ }^{2}$ Sarcoma Patients Euronet, D-85521 Riemerling, Germany
} 


\begin{abstract}
(Continued from previous page)
but translation of subjective experience into an objectively determined judgment is imperfect.

Quality of life and PROs stood out as a critical development area. In research there is no coherent vision but a lot of scattered activity, albeit with active consensus groups among academic researchers. Patient involvement in this area of research needs to develop further and become more effective. The idea that you can have Patient Reported Outcomes without patient provided inputs to inform the methods and processes used, is irrational and probably unethical. I propose that a vision based on defining patient pathways informed by PROs which patients have been involved in developing would underpin a life quality description which patients can easily relate to and which doctors would find easy to communicate. Pathways would also offer value to regulators and commissioners by giving them a focus on how clinical activity delivers patient benefit.
\end{abstract}

Keywords: Quality of life, QoL, HrQoL, Healthcare related quality of life, PROs, Patient reported outcome measures, PROMS, Cancer research, Patient involvement, Patient benefit, Survivorship, All trials, End-of-life

\section{Background}

Patient involvement is about adding value through the application of experience combined with the ability to stand back, bringing a subjective view, rather than offering an objective technical assessment. Patients come at research questions from a different direction than professionals and they can use that to open eyes to challenges which seem obvious once identified. After 15 years as an involved patient in cancer research I realized that I had acquired a healthy view of the realities, with some scepticism, some ambition and a readiness to see past the hype. When I started looking around I found I was asking myself, where is the next leap in real patient benefit going to come from? I came to the conclusion that PROMs, Patient Reported Outcome Measures, are where real progress can be made. The greatest potential benefit for the majority of patients, whether survivors or not, will be through addressing quality of life issues. Patients are involved in research in various ways but not as much in the development of PROs as they should be. So the purpose and practice of quality of life research should lead to patient benefit and that is more likely to happen if patients are involved.

\section{Research}

Dr. Ian Tannock gave a keynote talk at the National Cancer Research Institute (NCRI) Conference in 2014 on randomized controlled trials (RCTs), the gold standard for new therapies in oncology [1]. His team reviewed RCTs in breast, lung and colon cancer published since 1975 in major journals. He had some stark findings (my summary):

- We are looking for smaller benefits AND we are ignoring older drugs which might do the same

- We are using surrogate endpoints which are difficult to analyse AND drug approvals are based on them

- Harmful side effects are under-reported BUT don't worry, they get identified after approval
- Author bias favours experimental arms AND publication bias favours positive results

- Quality of life (QoL) is under researched AND QoL methods are poor

As a detached appraisal this analysis suggests that data are garnished to claim fancy conclusions, a few weeks added life is hyped as a significant benefit while data on the outcomes patients worry about are missing.

For most of the years which Tannock reviewed, patient involvement in research was a rarity. Academic studies, which more commonly involve patients in their development processes, may be exempt from these criticisms, although this needs research. Tannock reviewed all published studies and during the time period covered patient involvement got underway. It is also significant that the majority of studies included were industry sponsored studies with a registration objective where patient involvement and even the study of the quality of life of patients was not usual until quite recently. More recent reviews of the US Food \& Drug Administration (FDA) and European Medicines Agency (EMA) approval decisions confirm this analysis [2, 3].

The direction of clinical cancer research is taking us away from big RCTs looking at small effects towards more targeted interventions based on biology and genetics where response is probable, although side effects and recurrence are still real issues. The -ibs and -abs have been with us since trastumuzab (Herceptin) and imatinib (Glivec) were trialled in the 1990s. These two classes of drugs have driven up the cost of treating cancer hugely, do not deliver a cure except in one or two very rare cases, and many carry a side-effect burden which their manufacturers try not to talk about. Professor Charlie Swanton's work at University College London is pointing towards evolutionary genetics in cancer helping determine downstream therapy (the TRACERx study) [4] and it can be argued that many of these targeted drugs may work best early in the disease when a single pathway mutation is causing the cancer. 
However clinical and regulatory structures discourage that from happening and initial clinical studies for most drugs are in metastatic disease. Among the few that are used early in the disease imatinib (Glivec) is prescribed at diagnosis for chronic myeloid leukaemia. It offers long term benefit and as well as being one of the first of these drugs it is one of very few significantly in profit for its manufacturer.

The current clinical research headline themes are immunology, genetics and personalised medicine. Immunology is the latest extension of the procession of -ibs and -abs. There may be a cure for a few more cancers, there may not. We have no long term experience and side effects are a big issue. My concern is that in a year or two we may have a sense of deja vu.

Genomic medicine is the scientific wonder of the age. In cancer its goal is "understanding" the disease, opening the way to treating or curing it. Analysing the individual genome to map all the genomic variations (or mutations) will open up the route to precision treatment. It does not guarantee that any precise treatment will be available and we have already seen that cancer is adaptable. As far back as 2003 the mystery of why a primary gastro-intestinal stromal tumour (GIST) responded to imatinib while synchronous metastatic tumours did not, was unravelled. Patients have previously unknown mutations in their metastases [5] but metastases are rarely excised or biopsied limiting our ability to identify mutations. This supports the findings of Professor Swanton who was able to use new liquid biopsy techniques [6] not available to the earlier GIST study.

Precision medicine, or (inaccurately) personalised medicine, is the logical extension of this understanding. When a patient relapses after a treatment, knowing what mutations are now driving the cancer can lead to targeting them with further treatment regardless of the histology of the tumour. It means regular biopsies, using the new liquid biopsy techniques rather than core needle or surgical biopsy. It may also mean new side effects from the new treatment or additional side effects if the original treatment is still being taken because some tumours are still responding. Pharmacogenomics may lead to more targeted drug treatments or may identify side effects to which a patient is genetically predisposed. The prospect of patient burden is real and these risks are quite often not properly communicated. One risk in this concept is that a treatment for a previously unknown mutation may not exist or can not be produced in time to treat the patient.

Personalised medicine is starting to have doubters. A paper from September 2016 in Nature has a very telling line in it, "does the rhetoric so far outpace the reality that we risk fooling even ourselves?" [7].

Despite all the activity and the hype which has surrounded it, there are some cancers which are not benefiting from these advances. Predominant theories on the underlying cause of cancer see it as a cell-based disease. Is that the right approach? There is a hypothesis from respected researchers in the US and France arguing the case for a 'tissue organisation field theory' [8]. While this is not generally accepted is it possible that both theories are true, but in different cancers? We have to find answers but we still don't know all the questions we need to ask.

\section{Delivering treatment}

Treatment in local hospitals, in the UK district general hospitals (DGH), is the heartland where patient benefit has to be realised. It is easy for patients focused on involvement in research to forget this fact. Cancer centres in university hospitals, which predominate in research, are very different environments from the routine of a DGH. Doctors work under different pressures, there is limited access to research studies, and few have interests which push at the boundaries of treatment and care.

In too many hospitals patients are still dying within 30 days of their last chemotherapy. In 2009 the National Confidential Enquiry into Patient Outcomes and Death (NCEPOD) [9] defined this measure for us. A recent follow up study by the National Cancer Registration and Analysis Sevice (NCRAS) [10] shows that practice in some places has hardly changed. How do oncologists support patients making decisions about chemotherapy? It is improving but a recently published analysis by a retired oncologist indicates that it is badly handled in too many cases [11]. A Dutch study [12] describes collusion between patient and physician when discussing chemotherapy. This collusion "may facilitate unrealistic optimism and unjustified hope". In addition doctors do not adequately explain prognosis. The concept of 'winning the battle', created by social pressures, reinforces acceptance of toxic therapy at the end of life. Consider the Temel study of palliative care in advanced lung cancer [13]. This was the US study where patients were randomised to standard treatment plus or minus proactive palliative care. The palliative care group had better quality of life, used fewer drugs and lived longer. This lesson seems to be fading from memory.

\section{Survival}

We must not overlook the fact that at ten years following diagnosis $50 \%$ of all cancer patients are surviving and this percentage will improve. Earlier diagnosis, more accurate diagnosis through better imaging and pathology, and new surgical and radiotherapy techniques are all contributing. The inarguable fact is that in many cancers a cure is achieved through primary treatment, most frequently involving surgery.

There is a new emphasis on 'survivorship' in which self-management and quality of life appraisal have key 
roles to play [14]. The area is generally under-researched and patient experience is not described in ways which allow 'pathways' through survival to be defined. The initiative of the National Cancer Research Institute (NCRI) working with the James Lind Alliance (JLA) [15] to address the priorities for research into 'living with and beyond cancer' [16] is to be welcomed. It will involve a very wide ranging group of patients at all stages of the programme of work.

\section{The role of PROs}

Quality of life appraisal in research is not integrated and it lacks a coherent vision. Tannock made the point that QoL is under-researched and QoL methods are poor. A recent systematic review suggests that awareness among cancer research clinicians remains poor [17]. There are too many tools and no clarity on how any of them can lead to changes in clinical practice. There is a great variation in the quality of implementation and overall standards need to be consistently higher. If we unpack it we find:

- A multitude of tools - a Google search identified 18 used in cancer, there are certainly more, ranging from generic to disease specific

- Criteria are defined by academics and doctors not by patients - few tools mention patient involvement in papers describing their development and maintenance

- Data which are cherry-picked to support a pre-determined clinical or commercial view - a concern identified by systematic reviews $[2,18]$ and expressed verbally to me by regulatory officers.

- Piles of data which cannot be compared or aggregated - even assuming they are published or made available

- After 20 years we cannot describe a life-quality pathway through any cancer

The scarcity of published data on these issues contrasts with my experience listening to researchers, regulators, commissioners and patient groups. Working on development of guidance I have more than once been struck by the fact that the story told by a research publication contrasts with the reported experience of clinicians using that same drug in clinical practice. This is confirmed in a 2017 BMJ Editorial [19].

The efforts of the All Trials initiative to get all clinical studies, past, present and future, published in full are to be welcomed. There are moves involving journal editors to develop standardized approaches to reporting research. Adverse event data must be published, not hidden away, and QoL data must not be sidelined because it produces results which conflict with the desired outcomes. Most academic and charitable sponsors/funders have committed to support the All Trials principles [20].

At this point it will be valuable to clarify two terms. A Healthcare related Quality of Life assessment (HrQoL) is a structured multi-item assessment using data provided by patients but processed within a methodology to deliver a metric, some form of score. It may cover direct experience of disease or treatment but will also include specific questions which are important to the condition experienced by the patients. These may include physical, social or psychological parameters. The broader term Quality of Life (QoL) will also include factors beyond healthcare and is used in this paper to include all aspects of a patient's life. Patient Reported Outcomes (PROs) are a report coming directly from patients on a specific issue without interpretation describing how they function or feel in regard to a condition or therapy [21]. The term includes a wide range of constructs and methodology and can encompass concepts from symptoms to physical function, well-being and social involvement. The process may be described as a Patient Reported Outcome Measure (PROM).

The problems that researchers face in implementing an effective QoL element in their research have been described in a systematic review (2014) of available PRO guidance (not specific to cancer):

PRO-specific protocol guidance is difficult to access, lacks consistency and is unwieldy; with over 160 recommendations spread across 54 different publications. It is therefore extremely challenging to implement in practice [22].

Both the European Medicines Agency (EMA) [23, 24] and the US Food \& Drug Administration [25] (FDA) have attempted to address these issues in their guidance to researchers. Their focus is primarily on drugs seeking registration. The EMA 2005 paper even indicates the acceptability of an HrQoL endpoint and an efficacy endpoint as co-primary endpoints. The FDA guidance also highlights the importance of the role of patients to inform PRO content and improve validity and relevance to the patients in the study.

In the last twenty years QoL research has come a long way and progress since the EMA and FDA papers (2005 and 2009 respectively) has been substantial, However the core criticisms indicated above remain largely unaddressed.

I believe that the biggest impact on patient benefit which patients involved in research can enable is through involvement in the development of structured quality-of-life assessment and PROs which explore the detail of patient experience. The experience focus must be on studies into any kind of diagnostic approach, treatment, survival and importantly palliative and end-of-life 
care. The idea that you can have Patient Reported Outcomes without patient provided inputs to inform the methods and processes used, is irrational and probably unethical. As involved patients we need to move actively beyond patient involvement to becoming patients with a clear purpose and influence in the design process [26]. We, by that I mean established patient advocates/representatives, must use our influence to ensure that recent patients, the ones whose experience is fresh and hurting, actually define content and make decisions. We can be involved in direction and oversight but we must recognise that we may not be the right patients to do the defining. The further role of the experienced involved patients is to ensure that PROMs data are analysed and are published, ideally within the main study publication. Recognising that there are these twin levels of involvement will make an impact on patient benefit.

\section{Influencing cancer care}

We need to move quality-of-life research on, to become a new centre of influence in cancer care. The first priority is that we must have a single, consistent quality of life approach. The data must be produced and analysed in a way which facilitates comparison between studies and must be capable of being aggregated so that pathways of care can be described from a patient viewpoint.

Development of QoL in this way in cancer is happening in the UK, but not in research. Following the recommendations of the National Cancer Taskforce [27] the creation of a national HrQoL metric for NHS England's Cancer Dashboard is underway, although how this will impact on clinical practice remains unclear. The Dashboard is designed to inform at a generic level rather than aiding individual patient decision-making [28] but individual data will be gathered to create the overall dataset. A pilot project started in late 2017 gathering data from a small number of sites in three of the commoner cancers. Full implementation is planned for 2019. Macmillan Cancer Support is involved and is also behind another important piece of work - the Holistic Needs Assessment (HNA), part of its so-called Recovery Package derived from the work of the National Cancer Survivorship Initiative. The HNA underpins supportive non-medical care for cancer patients and is slowly being rolled out in the NHS [29]. Critically it is building experience among clinicians and patients about using electronically delivered patient questionnaires in cancer clinics, something which will become ubiquitous in the UK [10].

I would like to see a rounder understanding of what quality of life means to patients. Quality-of-life for the Cancer Dashboard is a structured assessment, the Holistic Needs Analysis uses PROs to define non-medical patient needs, and new PROs could fill in the gaps between the two. The whole seen together would would be much more than clinical. It would also inform such people as counsellors, social care providers and end-of-life care teams about the patient, complementing what they may be saying about expectations and preferences. Such an approach could bring research, routine clinical healthcare and social care together. PROs (single factor assessments) and quality of life (structured multi-factor assessment) would work together and deliver benefits from a well grounded perspective.

\section{Building new purpose for PROMs}

So what is actually happening in the development of HrQoL and PROs for use in cancer research? Is there a vision which is wider than the UK? A review of the PROs scene reveals that activity across the whole of healthcare is growing and the use of HrQoL data to support policy development, regulatory decision-making, commissioning and treatment is happening. Patients sit on many of these panels and want to hear about the patient experience. There is also a growth in patient involvement in developing PROs across a wide range of diseases. In cancer there are a number of initiatives, some relatively small and some with patients actively involved.

The European Organisation for the Research and Treatment of Cancer (EORTC) is in the forefront. Its SISAQOL project (Setting International Standards in Analyzing Patient-Reported Outcomes and Quality of Life Endpoints Data) [30] has brought together worldwide academic QoL experts, clinicians and patient activists, to look at developing an international set of data standards enabling better analysis of QoL/PROM data gathered in cancer research. It is early days but the European Medicines Agency and the US Food \& Drug Administration are supporting it and they would be the engines for changing the pharma industry.

The University of Birmingham has launched the Centre for Patient Reported Outcome Research (CPROR) [31]. The range of collaborators is world class. Where EORTC is looking at standards CPROR is looking at the actual PROs and the guidance available to study developers. They are working alongside each other. CPROR has been involved in the development of CONSORT-PRO, the patient reported outcome extension of the CONSORT standards. It is also running the SPIRITPRO extension project. CONSORT is a collaborative consensus between clinical trial methodologists, guideline developers, knowledge translation specialists and journal editors with the aim of improving randomised clinical trial reporting. The CONSORT PRO Extension [32] gives guidance to those developing randomised studies with PROs in their design as primary or secondary endpoints. SPIRIT-PRO is addressing the 
weakness of guidance available to trial developers using PROs through a Delphi project with over 100 experienced trial developers. CPROR also has the objectives of building capacity in applied PRO research and supporting researchers, funders and regulatory bodies to deliver high quality PROs data through both research projects and routine care.

The importance of aggregating and comparing PROM data has been recognized by an international group known as COSMIN, COnsensus-based Standards for the selection of health Measurement Instruments [33]. They provide a methodology for systematic reviews as well as a checklist approach offering guidance to researchers. The COMET initiative [34] brings together researchers developing standardised 'core outcome sets' which can be used in randomised clinical trials and clinical audit so that data can be gathered and analysed in a common way in any disease, improving comparability between studies.

The International Society for Quality of Life Research (ISOQOL) is a partner in the CONSORT and SPIRIT projects and members of all the above mentioned projects are also members of ISOQOL. Another member is Warwick Research in Nursing (WRIN) Warwick Medical School two of whose core workstreams are around patient and public involvement (PPI) and patient reported outcomes (PROs). They have worked to convince ISOQOL members of the value of patient involvement and engagement [35] and this is now set out clearly in a supportive statement to ISOQOL's vision [36].

For the UK an important agent in all of this is NICE, the National Institute for Health \& Care Excellence. They are working with Sheffield's School of Health and Related Research (ScHARR) to improve the methods by which they translate QoL data into the calculation of the ICER, the Incremental Cost Effectiveness Ratio, which underpins the health economics in Technology Appraisals. There are other prominent UK groups working on quality of life related projects. The team in Leeds has been using information technology to gather data at the front-line of healthcare in a number of very practical trials. In Oxford the Quality and Outcomes of Person-centred Care Policy Research Unit (QORU) has a programme informing policy-makers and commissioners.

While there are many parallel themes I do not see a coherent vision, the kind of integrating clarity that we see in the Cancer Dashboard project. The historical fragmentation in QoL referred to earlier is not being effectively challenged by these consensus groups, and many of these groups have common members. There has so far been little impact in cancer although in other, perhaps less complex, disease areas there has been good progress. The heterogeneity of cancer does not lend itself readily to generic approaches so I shall be following the Dashboard project with great interest. However we need something more, evidence in each of the disparate cancers which offers benefit to patients, both directly through providing reliable information and advice, and indirectly through influencing regulatory decisions, access to treatments, funding and commissioning.

\section{Conclusion - the pathway concept}

Describing the patient pathway seems to me to be common sense. It could provide a vision which all could work towards, using disparate skills, but by working together to unlock patient benefit and a lot more besides. At the centre of any development of this kind must be patients themselves, it makes no sense at all to be researching Patient Reported Outcomes unless patients are providing Inputs to that research, the risk of irrelevance by failing to do so is high.

We need to measure and describe the pathways experienced by patients in terms that patients understand. This would be done by bringing together quality of life data from a range of clinical and research sources, aggregating and analyzing it, to describe stages in the disease pathway. Add the relevant medical and clinical information, do this along the patient journey, and you have a series of descriptions which can underpin the understanding of clinical care and treatment. Many of the developments noted in this commentary would fit into this concept with little or no modification. This concept provides a unifying purpose.

Its impact in the wider area of clinical research would be that the study of a new treatment would have to demonstrate its value in the pathway relevant to that disease, not at a single isolated point in time. Clinical outcome measures may become secondary to quality-oflife outcomes in some cases. The way would be opened for longitudinal studies using PROs within a reference structure (the pathway), interpreted by patients rather than with, more arbitrary, researcher-defined outcomes. The existence of pathways would provide frameworks for developing new research ideas. Areas of clinical need would stand out and demand attention. As patients progress along a pathway we would be able to inform them much more fully about the risks and benefits to expect when they consider a treatment, whether that is the standard therapy or a new intervention.

A well-described evidence based pathway is a good vehicle for identifying risks within a recognizable context. If a risk is realized a new pathway or branch would open up and consequences, mitigation interventions etc. would be described and could be further researched.

The pathway will bring together disparate data and clarify situations which currently are open to variable 
individual interpretation. It adds value in a patientcentred and patient-sensitive way to what we have now. The potential benefits are huge:

- Better information for both patients and clinicians

- Studies would no longer record outcomes in isolation

- PROs could be the primary outcomes in research more easily

- Smaller effect sizes in studies would have context

- Smaller studies would be valid - creating huge savings in time and cost

- We would see fewer 'look-alike' and 'new for the sake of it' drugs

- It would be impossible to hide treatment side effects

- Innovation would be encouraged eg the recent collaboration between a large pharma company and the NCRI Consumer Forum [37]

- Regulators would trust data and could manage interim approvals more easily

- Ineffective treatments would be identified quickly

This pathway approach may facilitate the appraisal of treatments defined as end-of-life. NICE currently utilizes a higher ICER threshold for approving such treatments even when there is only a small improvement in duration of life. A reliable pathway picture, demonstrating the QoL of patients, would help everybody involved.

It might appear that elements for the pathway concept are falling into place but it is not in any integrated kind of way. The unifying vision is missing. The challenge is to get co-ordination and collaboration into place, to create and sustain an integrated vision which drives standardisation of PROs and quality of life in a way which is relevant to patients. This move would add pressure for PROs to be adopted more usually as a primary outcome measure in cancer clinical research. I believe such a collaboration to develop pathways of care and treatment underpinned through quality-of-life measures must be supported and enabled by effective patient engagement and involvement. One of our roles as patients is to be the 'keeper of the vision', not to be technical authors. We can ensure directions are maintained, provide insights, and we can offer valid and useful input as the development progresses.

This is a view being articulated by a patient. No-one I have spoken to thinks it is unreasonable but is anyone prepared to respond and take the lead? At the very least we should have a debate. I believe the pathway concept needs effective patient influence if it is to happen, even patient leadership, but it also needs an independent organization with influence to host it and there will be a requirement for investment.

\section{Abbreviations}

COMET: Core Outcome Measures In Effectiveness Trials; COSMIN: Consensus based Standards for the selection of health Management Instruments; CPROR: Centre for Patient Reported Outcomes Research; DGH: District General Hospital; EMA: European Medicines Agency; EORTC: European Organisation for the Research and Treatment of Cancer; FDA: US Food \& Drug Administration; HNA: Holistic Needs Analysis; ICER: Incremental Cost Effectiveness Ratio; ISOQOL: International Society for Quality of Life Research; JLA: James Lind Alliance; NCEPOD: National Confidential Enquiry into Patient Outcome and Death; NCRAS: National Cancer Registration and Advisory Service; NCRI: National Cancer Research Institute; NICE: National Institute for Health and Care Excellence; PPI: Patient and Public Involvement;

PROMs: Patient Reported Outcome Measures (also PROs); QoL: Quality of Life - also HRQoL - Health Related Quality of Life; QORU: Quality and Outcomes of Person-centred Care Policy Research Unit; RCT: Randomised controlled trial; RCT: Randomised Controlled Trial; ScHARR: School of Health and Related Research; SISAQoL: Setting International Standards in Analyzing PatientReported Outcomes and Quality of Life Endpoints Data; WRIN: Warwick Research in Nursing, Warwick Medical School

\section{Acknowledgements}

Roger Wilson acknowledges the NCRI Consumer Forum for their support and encouragement while he developed this commentary.

Funding

Not applicable.

Availability of data and materials

Not applicable.

Authors' contributions

$\mathrm{RW}$ is the sole author.

\section{Authors' information}

Roger Wilson was diagnosed with a sarcoma in 1999 and has had six recurrences since then. He has been involved in cancer research since 2002 and has chaired and worked with a number of national and international projects and groups over the years. He is a member of the NCRI Consumer Forum, the founder of Sarcoma UK and co-founder of Sarcoma Patients Euronet. He was awarded the CBE in the New Year Honours 2011 for services to healthcare.

Ethics approval and consent to participate

Not appllcable.

Consent for publication

Not applicable.

\section{Competing interests}

The author declares that he has no competing interests.

\section{Publisher's Note}

Springer Nature remains neutral with regard to jurisdictional claims in published maps and institutional affiliations.

Received: 24 November 2016 Accepted: 8 January 2018

Published online: 26 February 2018

References

1. Iain Tannock, Princess Margaret Hospital, Toronto, Ontario, Canada: Evolution of the cancer clinical trial over three decades: problems of design, analysis, reporting and interpretation. http://www.downyoutubeinmp4.com/ watch?v=4f1y2flw1jo. Accessed 28 Jan 2018.

2. Davis C, Naci H, Gurpinar E, et al. Availability of evidence of benefits on overall survival and quality of life benefits of cancer drugs approved by the European medicines agency: retrospective cohort study of drug approvals from 2009-2013. BMJ. 2017;359:j4530.

3. Kim C, Prasad V. Cancer drugs approved on the basis of a surrogate end point and subsequent overall survival: an analysis of 5 years of US Food and Drug Administration approvals. JAMA Intern Med. 2015;359:1992-4. https:// doi.org/10.1001/jamainternmed.2015.5868. pmid:26502403 
4. Mariam Jamal-Hanjani, Gareth A. Wilson ..... Allan Hackshaw, and Charles Swanton, for the TRACERx Consortium. Tracking the Evolution of NonSmall-Cell Lung Cancer. England J Med, 2017 DOI: https://doi.org/10.1056/ NEJMoa1616288.

5. Prasad V. Perspective: the precision-oncology illusion. Nature. 2016;537:S63. https://doi.org/10.1038/537S63a. Published online 07 September 2016

6. Abbosh C, Birkbak NJ, Wilson GA, Jamal-Hanjani M, et al. Phylogenetic ctDNA analysis depicts early-stage lung cancer evolution. Nature. 2017:545: 446-51. https://doi.org/10.1038/nature22364. Published online 26 April 2017

7. Serrano C, Wang Y, et al. KRAS and KIT gatekeeper mutations confer polyclonal primary Imatinib resistance in GI Stromal tumors: relevance of concomitant Phosphatidylinositol 3-Kinase/AKT Dysregulation. J Clin Oncol. 2015;33(22):e93-6.

8. Soto AM, Sonnenschein C. The tissue organization field theory of cancer: a testable replacement for the somatic mutation theory. BioEssays. 2011;33(5): 332-40. https://doi.org/10.1002/bies.201100025.

9. A report published by NCEPOD. For better, for worse? A review of the care of patients who died within 30 days of receiving systemic anti-cancer therapy. London: The National Confidential Patient Outcome and Death 2008.

10. Wallington M, Saxon EB, Bomb M, Smittenaar R, Wickenden M, McPhail S, et al. 30-day mortality after systemic anticancer treatment for breast and lung cancer in England: a population-based, observational study. The Lancet Oncology. 2016;17(9):p1203-16 Published: September.

11. Wise P. Cancer drugs, survival and ethics. BMJ. 2016:355:i5792

12. van Dijk EF, Coşkuntürk M, Zuur AT, van der Palen J, van der Graaf WT, Timmer-Bonte JN, Wymenga AN. Willingness to accept chemotherapy and attitudes towards costs of cancer treatment; a multisite survey study in the Netherlands. Neth J Med. 2016;74(7):292-300.

13. Temel JS, et al. Early palliative Care for Patients with metastatic non-smallcell lung cancer. N Engl J Med. 2010;363:733-42. https://doi.org/10.1056/ NEJMoa1000678.

14. Richards M, Corner J, Maher J. The National Cancer Survivorship Initiative: new and emerging evidence on the ongoing needs of cancer survivors. $\mathrm{Br}$ Cancer. 2011;105(Suppl 1):S1-4. https://doi.org/10.1038/bjc.2011.416.

15. James Lind Alliance website. http://www.jla.nihr.ac.uk/about-the-james-lindalliance/ Accessed 4 Oct 2017.

16. NCRI Living With and Beyond Cancer Research Initiative - April 2017 http:// www.ncri.org.uk/what-we-do/initiatives/lwbc/ accessed 12 June 2017.

17. King S, Exley J, et al. The use and impact of quality of life assessment tools in clinical care settings for cancer patients, with a particular emphasis on brain cancer: insights from a systematic review and stakeholder consultations. Qual Life Res. 2016;25:2245-56. Published online 2016 Apr 2. https://doi.org/10.1007/s11136-016-1278-6.

18. Schandelmaier S, Conen K, von Elm E, DISCO study group. Planning and reporting of quality-of-life outcomes in cancer trials. Ann Oncol. 2015;26(9): 1966-73. https://doi.org/10.1093/annonc/mdv283. Epub 2015 Jun 30

19. Prasad V. Do cancer drugs improve survival or quality of life? BMJ. 2017;359: j4528. https://doi.org/10.1136/bmj.j4528. (Published 04 October 2017)

20. All Trials website. http://www.alltrials.net/ Accessed 4 Oct 2017.

21. Donald L. Patrick, Laurie B. Burke, John H. Powers, Jane A. Scott, Edwin P. Rock, Sahar Dawisha, Robert O'Neill, Dianne L. Kennedy .Patient-reported outcomes to support medical product labeling claims: FDA perspective. Value Health. 2007;10(Supplement 2):S125-37.

22. Calvert M, Kyte D, Duffy H, Gheorghe A, Mercieca-Bebber R, Ives J, et al. Patient-reported outcome (PRO) assessment in clinical trials: a systematic review of guidance for trial protocol writers. PLoS One. 2014;9(10):e110216. https://doi.org/10.1371/journal.pone.0110216

23. Committee for Medicinal Products for Human Use (CHMP). Reflection paper on the regulatory guidance for the use of Healthrelated quality of life (Hrql) measures in the evaluation of medicinal products. London: EMA; 2005. Doc. Ref. EMEA/CHMP/EWP/139391/2004

24. Appendix 2 to the guideline on the evaluation of anticancer medicinal products in man: EMA Committee for Medicinal Products for Human Use (CHMP): 1 April 2016: EMA/CHMP/292464/2014.

25. Guidance for Industry Patient-Reported Outcome Measures: Use in Medica Product Development to Support Labeling Claims: U.S. Department of Health and Human Services Food and Drug Administration: December 2009.

26. Kirstie L. Haywood, Roger Wilson, Sophie Staniszewska, Sam Salek. Using PROMs in healthcare: who should be in the driving seat-policy makers, health professionals, methodologists or patients?. Patient. 2016. doi:https:// doi.org/10.1007/s40271-016-0197-5.

27. Achieving World-Class Cancer Outcomes: Taking the strategy forward NHS England Publications Gateway Reference 05215.

28. Public Health England - Cancer Dashboard - at Phase 1 of development on Internet https://www.cancerdata.nhs.uk/dashboard\#?tab=Overview Accessed 4 June 2017.

29. Evaluation of the Electronic Holistic Needs Assessment (eHNA) Final Evaluation Report. IPSOS-Mori for Macmillan Cancer Care September 2015.

30. Andrew Bottomley et al, for the Setting International Standards in Analyzing Patient-Reported Outcomes and Quality of Life Endpoints Data (SISAQOL) consortium. Analysing data from patient-reported outcome and quality of life endpoints for cancer clinical trials: a start in setting international standards. Lancet Oncol. 2016 DOI: https://doi.org/10.1016/S14702045(16)30510-1.

31. CPROR website: http://www.birmingham.ac.uk/research/activity/mds/ centres/cpror/index.aspx Accessed 20 June 2017.

32. Calvert M, Blazeby J, Altman DG, et al. Reporting of patient-reported outcomes in randomized trials: the CONSORT PRO extension. JAMA. 2013; 309(8):814-22. PMID: 23443445

33. Mokkink LB, Terwee CB, Patrick DL, Alonso J, Stratford PW, Knol DL, Bouter $\mathrm{LM}$, de Vet HCW. International consensus on taxonomy, terminology, and definitions of measurement properties: results of the COSMIN stud. J Clin Epidemiol. 2010:63:737-45.

34. COMET website: http://www.comet-initiative.org/about/overview Accessed 20 June 2017.

35. Haywood K, Brett J, Salek S, Marlett N, Penman C, Shklarov S, Norris C, Santana MJ, Staniszewska S. Patient and public engagement in healthrelated quality of life and patient-reported outcomes research: what is important and why should we care? Findings from the first ISOQOL patient engagement symposium. Qual Life Res. 2015;24(Issue 5):1069-76. https:// doi.org/10.1007/s11136-014-0796-3.

36. ISOQOL website: mission statement: http://www.isoqol.org/about-isoqo Accessed 20 June 2017.

37. The NCRI Consumer Forum Dragon's Den - including a 'hackathon' http:// www.ncri.org.uk/newsletter-2/latest-news-from-the-ncri-consumer-forum-2/ Accessed 20 June 2017

\section{Submit your next manuscript to BioMed Central and we will help you at every step:}

- We accept pre-submission inquiries

- Our selector tool helps you to find the most relevant journal

- We provide round the clock customer support

- Convenient online submission

- Thorough peer review

- Inclusion in PubMed and all major indexing services

- Maximum visibility for your research

Submit your manuscript at www.biomedcentral.com/submit
) Biomed Central 